\title{
Lifetime economic burden of prostate cancer
}

\author{
Michael E Stokes ${ }^{1 * \dagger}$, Jack Ishak ${ }^{1 \dagger}$, Irina Proskorovsky ${ }^{1 \dagger}$, Libby K Black $^{2+}$ and Yijian Huang ${ }^{3+}$
}

\begin{abstract}
Background: Prostate cancer (PCa) is the most common cancer affecting men in the United States. The initial treatment and subsequent monitoring of PCa patients places a large burden on U.S. health care systems. The objectives of this study were to estimate the total and disease-related per-patient lifetime costs using a phasebased model of cancer care for PCa patients enrolled in Medicare.

Methods: A model was developed to estimate life-time costs for patients diagnosed with PCa. Patients $\geq 65$ years old and diagnosed with PCa between calendar years 1991-2002 were selected from the SEER database. Using SEER, we estimated survival times for PCa patients from diagnosis until death. The period of time patients contributed to treatment phases was determined using an algorithm designed to model the natural history of PCa. Costs were obtained from the US SEER-Medicare database and estimated during specific phases of care. Cost estimates were then combined with survival data to yield total and PCa-related life-time costs.
\end{abstract}

Results: Overall, the model estimated life-time costs of $\$ 110,520$ (95\% Cl 110,324-110,739) per patient. PCa-related costs made up approximately $31 \%$ of total costs $(\$ 34,432)$.

Conclusions: Prostate cancer places a significant burden on U.S. health-care systems with average life-time PCarelated costs in excess of $\$ 30,000$.

Keywords: Cost model, lifetime costs, prostate cancer, Managed care, Medicare

\section{Background}

According to the National Cancer Institute's Surveillance Epidemiology and End Results (SEER) database, prostate cancer (PCa) prevalence in 2008 was estimated at $2,555,936$ [1]. Prostate cancer is the most common cancer affecting men in the United States (U.S.) [1]. This is a result of both advances in screening following introduction of the prostate specific antigen (PSA) test as well as increases in survival attributable to more effective therapies [2]. Although incidence has leveled off in recent years, the American Cancer Society estimates that approximately 186,000 men were diagnosed with PCa in 2008 [2]. Approximately $60 \%$ of incident prostate cancer cases are diagnosed at $\geq 65$ years of age [3]. The initial treatment and subsequent monitoring of these large numbers of PCa cases places a burden on U.S. health care systems. Prevalence based treatment costs for 2006 alone have been estimated at $\$ 9.6$ billion [4].

\footnotetext{
*Correspondence: michael.stokes@unitedbiosource.com

† Contributed equally

'Health Economics, United BioSource Corporation, 185 Dorval Avenue,

Montreal, Quebec, H9S 5J9, Canada

Full list of author information is available at the end of the article
}

Two therapies including finasteride and dutasteride have recently been studied for the prevention of $\mathrm{PCa}[5,6]$. Economic burden of illness studies are important because they quantify the costs that could be avoided should these pharmacologic agents prove effective in reducing incident cancer cases. Common approaches to estimating diseaserelated costs often include the use of a healthcare claims database. However, databases often do not provide enough years of follow-up to estimate life-time costs. This is particularly problematic for $\mathrm{PCa}$ as five-year survival rates approach $100 \%$ and censored cost data become a concern [1]. Thus, there is a paucity of data concerning life-time PCa costs. This study provides estimates of total and disease-related per-patient lifetime costs using a phase-based model of cancer care among patients diagnosed with prostate cancer at $\geq 65$ years of age.

\section{Methods \\ Model Overview}

We developed a phase-based model utilizing similar methodology as a prior study and applied it to PCa in order to estimate life-time total and disease-related costs for patients diagnosed with PCa [7]. Using survival data

\section{Biomed Central}


from SEER, we estimated survival times for PCa patients from diagnosis until death. The period of time that patients contributed to clinically relevant phases of treatment (initial, continuing, and terminal care) was then determined using an algorithm designed to model the natural history of PCa [7]. Finally, phase-specific monthly costs were combined with data on the number of months that patients were alive during each phase of care to yield total and PCa-related life-time costs. Analyses were conducted from a Medicare payer perspective.

\section{Patient Population}

Survival following PCa diagnosis was estimated using data from the SEER program. At the time of analyses, SEER was collecting data on cancer incidence, survival, and prevalence from specific geographic areas representing approximately $26 \%$ of the U.S. population [8]. Male patients $\geq 65$ years old with a first primary diagnosis of PCa between January 1, 1991 and December 31, 2002 were selected for inclusion from SEER using the cancer site recode variable. Patients were excluded if their cancer stage at diagnosis could not be identified or was Stage 0 .

\section{Estimation of Survival and Length of Treatment Phases}

Survival data from SEER were used to develop a statistical model designed to predict life expectancy for patients with Stage IV PCa. Time to death was calculated as the number of months between the date of index PCa diagnosis and the date of death or end of follow-up. Patients were censored if they were alive at the end of follow-up. Various parametric distributions including the exponential, Weibull, log-normal, and log-logistic were fitted to the data. Models were created with time to death as the response variable and continuous age as the independent variable. The log-normal distribution was chosen after comparing -2log-likelihood statistics for each model and comparing the median observed and predicted survival times to determine the best fitting distribution. Continuous age was included as a predictor in the models. A predictive equation was then derived from the model to estimate life expectancy for each Stage IV patient according to their age at diagnosis.

The majority of Stage I-III patients were still alive as of the last date survival data were collected in SEER. Therefore, it was not feasible to create a valid model to predict survival for these patients. For patients with no recorded date of death, we assumed survival was comparable to the U.S. general population. This was a conservative assumption based on a study by Brenner and Arndt which estimated relative survival rates for localized/regional PCa that were above $100 \%$ as compared to the general population [9]. Data from U.S. life tables based on age-specific 2004 death rates were used to estimate survival according to age and race [10].
Following estimation of survival, the time period from diagnosis until death was divided into distinct phases of care (initial, continuing, and terminal) using an approach similar to prior studies $[7,11]$ For patients surviving $\geq 18$ months, the initial phase was defined as the first 6 months following prostate cancer diagnosis, the terminal phase as the last 12 months prior to death, and the continuing care phase as the time in months between initial and terminal phases. For patients surviving $<18$ months, the final 12 months of follow-up were allocated first to the terminal phase and the remaining months were defined as initial phase.

\section{Estimation of Monthly Treatment Phase Costs}

Medicare payment data from the SEER-Medicare database was used to estimate monthly phase-specific total and disease-related medical costs [12]. Cost data were estimated from Medicare claims data spanning calendar years 1991 through 2004 for PCa cases diagnosed between January 1, 1991 and December 31, 2002 and were standardized to 2004 U.S. dollars. The main initial therapeutic options including prostatectomy, radiation, and hormone therapy were in use during the study periods over which Medicare costs were estimated. Monitoring for cancer recurrence with prostate specific antigen was also in practice.

Monthly treatment phase total and PCa-related costs that were input into the model are depicted in Table 1. PCa-related costs were estimated from the SEER-Medicare data using a sample of male patients from a random $5 \%$ sample of Medicare beneficiaries without cancer, matched to $\mathrm{PCa}$ cases on 5-year age groups. This non-cancer cohort was used to estimate the background medical costs unrelated to PCa. The incremental differences in the average monthly treatment phase costs between PCa patients and the cohort without cancer were defined as PCa-related costs.

Table 1 Mean Monthly Cost Estimates, by Stage and Treatment Phase for PCa Patients

\begin{tabular}{cccc}
\hline Study Measure & \multicolumn{3}{c}{ Treatment Phase } \\
\cline { 2 - 4 } & Initial Care & Continuing Care & Terminal Care \\
\hline Total costs & & $\$ 693$ & \\
Stage I & $\$ 2,058$ & $\$ 616$ & $\$ 3,167$ \\
Stage II & $\$ 2,333$ & $\$ 542$ & $\$ 3,044$ \\
Stage III & $\$ 2,774$ & $\$ 790$ & $\$ 3,056$ \\
Stage IV & $\$ 2,665$ & & $\$ 4,315$ \\
PCa-related costs & & $\$ 247$ & \\
Stage I & $\$ 1,615$ & $\$ 170$ & $\$ 37$ \\
Stage II & $\$ 1,890$ & $\$ 96$ & $\$ 0$ \\
Stage III & $\$ 2,331$ & $\$ 344$ & $\$ 1,185$ \\
Stage IV & $\$ 2,212$ & & \\
\hline
\end{tabular}




\section{Estimation of Life-time Total and PCa-related Costs}

Phase-specific monthly cost estimates were combined with survival data from SEER to calculate life-time costs for patients from diagnosis until death. For each patient, the corresponding phase-specific monthly cost was multiplied by the number of months patients contributed to each treatment phase.

\section{Data Analyses}

Baseline demographic and clinical characteristics including age, race, and geographic region were assessed descriptively by cancer stage for the PCa study cohort used to estimate survival from diagnosis until death. Life-time total and PCa-related costs were summarized by cancer stage. Confidence intervals were calculated around cost estimates using non-parametric bootstrap methods [13]. The study dataset was re-sampled with replacement to create 1,000 random samples. Total and prostate cancerrelated costs were then calculated for each of the 1,000 samples. Lower and upper confidence bounds were obtained from the sample distribution of costs at the $2.5 \%$ and $97.5 \%$ quantiles, respectively. Sources of variability accounted for in the bootstrap include costs according to treatment phase and cancer stage and the number of months that patients contribute to each treatment phase. Life-time total and PCa-related costs were discounted at a $3 \%$ annual rate.

Aggregate life-time treatment costs for U.S. incident PCa cases diagnosed in 2008 and $\geq 65$ years of age were calculated. By combining data from the American Cancer Society on the number of incident PCa cases $(186,620)$ diagnosed in 2008 with the proportion of cases $\geq 65$ years of age (60\%) from SEER statistics, we estimated the number of incident cases 65 years of age and older $(111,972)$ $[2,3]$. The number of incident cases was then multiplied by life-time treatment cost estimates in order to calculate the aggregate total and PCa-related burden of medical care among patients $\geq 65$ years old.

\section{Results}

\section{Patient Characteristics}

Patient characteristics are shown in Table 2. One-half (51.1\%) of patients were diagnosed at Stage I. Eighteen percent, $15.7 \%$, and $14.9 \%$ were diagnosed at Stages II, III, and IV, respectively. A higher proportion of black patients were diagnosed at Stage IV (14.0\%) compared to the overall proportion of black patients diagnosed in Stages I-III (10.0\%). The majority of patients $(57.4 \%)$ resided in the Western region at the time of $\mathrm{PCa}$ diagnosis. The predicted survival times from PCa diagnosis until death varied according to cancer stage (Table 2). Survival among Stage IV was shorter (Mean: 43.7 months, 3.7 years) versus Stages I-III (Mean: 178.2 months, 14.9 years). Patients diagnosed with Stage III PCa had longer mean predictive survival times compared to Stages I/II. The main driver of this result was that Stage III patients were diagnosed at younger ages (on average 2.3 years younger) versus Stages I/II (age at diagnosis: 71.3 vs. 73.6 years). The percentage of survival time Stage I-III patients spent in continuing care was higher $(90.4 \%$, 161.1 months) compared to Stage IV (66.8\%, 29.2 months).

\section{Total and PCa-related Per-Patient Life-time Costs}

Average per-patient total and PCa-related life-time costs are displayed in Table 3. For all patients, the model estimated average total life-time medical-care costs of $\$ 110,520$ (95\% CI: 110,324-110,739). PCa-related costs $(\$ 34,432,95 \%$ CI: 34,359-34,507) made up approximately $31 \%$ of total medical-care costs. Total medical costs ranged from \$73,587-\$120,085 (Stage I: \$120,085, 95\% CI: 119,819-120,357; Stage II: \$113,616, 95\% CI: 113,174114,067; Stage III: \$110,943, 95\% CI: 110,525-111,412; Stage IV: \$73,587, 95\% CI: 73,142-74,029). Stage IV total costs $(\$ 73,587)$ were lower reflecting shorter periods of survival compared to patients diagnosed at lower stages. PCa-related lifetime costs were highest for Stage I (\$39,182, 95\% CI: 39,075-39,292), followed by Stage II (\$31,915, 95\% CI: 31,769-32,063), Stage IV (\$30,038, 95\% CI: 29,824-30,244), and Stage III (\$26,078, 95\% CI: 25,975$26,189)$. However, after adjusting PCa costs by length of follow-up, per year costs for Stage IV $(\$ 8,118)$ were much higher relative to Stages I $(\$ 2,740)$, II $(\$ 2,128)$, and III $(\$ 1,590)$, reflecting higher medical resource use intensity characteristic of distant stage patients.

Aggregate life-time total costs for all incident cases $\geq$ 65 years and diagnosed in the U.S. during 2008 were $\$ 12.4$ billion. Aggregate costs associated with PCarelated medical care totaled approximately $\$ 3.9$ billion.

\section{Discussion}

Prostate cancer places a significant burden on the U.S. Medicare system with average per-patient life-time attributable costs of approximately $\$ 34,000$ (discounted at $3 \%)$. PCa-related costs represented approximately onethird of total medical care costs. Our analyses indicate that the aggregate life-time disease-related burden attributable to incident PCa cases $\geq 65$ years of age in 2008 approaches $\$ 4.0 \mathrm{~B}$ (discounted at 3\%). PCa-related lifetime costs were variable by cancer stage and ranged from $\$ 26,078$ (Stage III) to $\$ 39,182$ (Stage I). Stage IV had the poorest prognosis as the mean survival time was only 44 months compared to 172, 180, and 196 months for Stages I, II, and III, respectively.

A study conducted by Riley and colleagues examined total life-time costs of several cancers including prostate in elderly Medicare-eligible patients using the SEERMedicare database [14]. To the best of our knowledge 
Table 2 Demographic and Clinical Characteristics (SEER-Medicare, incident PCa cases $\geq 65$ years old diagnosed during 1991-2002)

\begin{tabular}{|c|c|c|c|c|c|c|c|c|c|c|}
\hline Characteristic & Stage I & & Stage II & & Stage III & & Stage IV & & All & \\
\hline $\mathrm{N}, \%$ & 71,861 & $51.1 \%$ & 25,590 & $18.2 \%$ & 22,103 & $15.7 \%$ & 21,011 & $14.9 \%$ & 140,565 & $100.0 \%$ \\
\hline \multicolumn{11}{|l|}{ Age at study index } \\
\hline Mean (SD) & 73.8 & (5.8) & 72.9 & $(5.8)$ & 71.3 & $(5.3)$ & 75.30 & $(7.2)$ & 73.5 & (6.1) \\
\hline Median & 73 & & 72 & & 70 & & 74 & & 73 & \\
\hline \multicolumn{11}{|l|}{ Age (5-year categories), N, \%: } \\
\hline $65-69$ & 18,974 & $26.4 \%$ & 8,715 & $34.1 \%$ & 9,738 & $44.1 \%$ & 5,424 & $25.8 \%$ & 42,851 & $30.5 \%$ \\
\hline $70-74$ & 22,922 & $31.9 \%$ & 7,844 & $30.7 \%$ & 7,378 & $33.4 \%$ & 5,401 & $25.7 \%$ & 43,545 & $31.0 \%$ \\
\hline $75-79$ & 18,140 & $25.2 \%$ & 5,486 & $21.4 \%$ & 3,230 & $14.6 \%$ & 4,349 & $20.7 \%$ & 31,205 & $22.2 \%$ \\
\hline $80-84$ & 8,235 & $11.5 \%$ & 2,428 & $9.5 \%$ & 1,177 & $5.3 \%$ & 3,261 & $15.5 \%$ & 15,101 & $10.7 \%$ \\
\hline $85+$ & 3,590 & $5.0 \%$ & 1,117 & $4.4 \%$ & 580 & $2.6 \%$ & 2,576 & $12.3 \%$ & 7,863 & $5.6 \%$ \\
\hline \multicolumn{11}{|l|}{ Race, N, \%: } \\
\hline White & 58,410 & $81.3 \%$ & 20,674 & $80.8 \%$ & 18,327 & $82.9 \%$ & 15,873 & $75.6 \%$ & 113,284 & $80.6 \%$ \\
\hline Black & 7,448 & $10.4 \%$ & 2,661 & $10.4 \%$ & 1,839 & $8.3 \%$ & 2,935 & $14.0 \%$ & 14,883 & $10.6 \%$ \\
\hline Asian & 2,572 & $3.6 \%$ & 916 & $3.6 \%$ & 807 & $3.7 \%$ & 861 & $4.1 \%$ & 5,073 & $3.6 \%$ \\
\hline Other & 3,431 & $4.7 \%$ & 1,339 & $5.2 \%$ & 1,130 & $5.1 \%$ & 1,342 & $6.3 \%$ & 7,325 & $5.2 \%$ \\
\hline \multicolumn{11}{|l|}{ Geographic region, N, \%: } \\
\hline Midwest & 14,040 & $19.5 \%$ & 4,531 & $17.7 \%$ & 4,216 & $19.1 \%$ & 4,357 & $20.7 \%$ & 27,144 & $19.3 \%$ \\
\hline Northeast & 12,303 & $17.1 \%$ & 3,416 & $13.4 \%$ & 2,041 & $9.2 \%$ & 2,426 & $11.6 \%$ & 20,186 & $14.4 \%$ \\
\hline South & 7,024 & $9.8 \%$ & 2,362 & $9.2 \%$ & 1,566 & $7.1 \%$ & 1,588 & $7.6 \%$ & 12,540 & $8.9 \%$ \\
\hline West & 38,494 & $53.6 \%$ & 15,281 & $59.7 \%$ & 14,280 & $64.6 \%$ & 12,640 & $60.2 \%$ & 80,695 & $57.4 \%$ \\
\hline Predicted mean survival time (SD) in months & 172 & $(85.9)$ & 180.1 & $(95.8)$ & 196.3 & $(107.0)$ & 43.7 & $(39.1)$ & 158.2 & $(99.2)$ \\
\hline \multicolumn{11}{|l|}{ Mean survival time (SD) in months } \\
\hline Initial & 5.8 & $(1.1)$ & 5.8 & (1.1) & 5.8 & $(1.1)$ & 4.4 & $(2.5)$ & 5.6 & (1.5) \\
\hline Continuing & 154.9 & $(85.0)$ & 162.9 & $(95.1)$ & 179.2 & $(106.2)$ & 29.2 & $(36.5)$ & 141.4 & (98.0) \\
\hline Terminal & 11.8 & $(1.2)$ & 11.8 & $(1.1)$ & 11.8 & $(1.7)$ & 10.6 & (3.1) & 11.6 & $(1.7)$ \\
\hline
\end{tabular}

$\mathrm{N}=$ Number of patients, $\mathrm{SD}=$ Standard deviation, SEER = Surveillance, Epidemiology, and End Results

this is the only study conducted to date that reports lifetime estimates of treatment costs. Riley et al. estimated costs in PCa patients $\geq 65$ years of age using data from 1984 to 1990 , an era pre-dating widespread use of the PSA screening test [14]. Comparison of our dataset with Riley et al. allows us to examine trends in survival and costs occurring over time. We report estimates of total life-time costs that are higher (Stages I, II: $\$ 118,261$, Stage III: $\$ 110,943$, Stage IV: $\$ 73,587)$ relative to Riley (Local: \$98,447; Regional: \$99,542; Distant: \$62,634, adjusted to 2004 US\$). We also estimate longer periods of survival (13.2 vs. Riley: 7.0 years). This finding is largely the result of the fact that the majority of $\mathrm{PCa}$ patients diagnosed in the era of widespread PSA screening that has taken place since the early 1990s are now diagnosed at earlier stages and no longer have excess mortality compared to the general population [9]. Upon adjusting total costs by year of follow-up, our estimates were actually lower $(\$ 8,373)$ relative to Riley et al. (\$13,028, adjusted to 2004 US\$) [14]. This finding may suggest that $\mathrm{PCa}$ patients can now expect to have lower morbidity related to earlier diagnosis and therefore also accrue fewer costs. Patients diagnosed at earlier stages may also be more likely to receive watchful waiting which has the effect of lowering costs relative to patients diagnosed at higher stages.

Table 3 Mean Life-time Costs and Survival, by Cancer Stage (US\$ 2004)*

\begin{tabular}{lccccc}
\hline Study Measure & Stage I & Stage II & Stage III & Stage IV & All Patients \\
\hline Number of patients & 71,861 & 25,590 & 22,103 & 21,011 & 140,565 \\
Total costs & $\$ 120,085$ & $\$ 113,616$ & $\$ 110,943$ & $\$ 73,587$ & $\$ 110,520$ \\
PCa-related costs & $\$ 39,182$ & $\$ 31,915$ & $\$ 26,078$ & $\$ 30,038$ & $\$ 34,432$ \\
Average years of survival & 14.3 & 15.0 & 16.4 & 3.7 & 13.2 \\
Total costs per year & $\$ 8,398$ & $\$ 7,574$ & $\$ 6,765$ & $\$ 19,888$ & $\$ 8,373$ \\
PCa-related costs per year & $\$ 2,740$ & $\$ 2,128$ & $\$ 1,590$ & $\$ 8,118$ & $\$ 2,608$ \\
\hline
\end{tabular}

${ }^{*}$ Costs discounted at $3 \%$ 
An interesting finding of our study was that Stage III PCa patients were diagnosed approximately 3.4 years younger compared to Stages I/II (age at diagnosis: 67.8 vs. 71.2 years). Prior studies examining treatment and survival outcomes using SEER registry data and data from a tumor registry in Germany have also similarly reported that Stage III patients are diagnosed at younger ages versus Stages I/II $[15,16]$. As part of our analysis, we assumed that survival among Stage I-III PCa patients would follow the U.S. general population based in part on 5-year relative survival statistics from SEER reporting that $\mathrm{PCa}$ patients diagnosed with local and regional disease is $100 \%$ [3]. Relative survival is a measure of net survival that is calculated by comparing overall survival with survival from similar individuals without cancer. We considered this to be a conservative assumption with respect to our estimation of costs since prior studies have reported relative survival for local/regional stage $\mathrm{PCa}$ in excess of $100 \%[9,16]$. This finding may be explained in part by the fact that many local/regional stage tumors are diagnosed as a result of PSA screening and men who undergo this preventive measure may in fact be healthier compared to men who do not participate in PSA testing [17]. Therefore, when we estimated predicted mean survival for Stage I-III patients based on U.S. life tables, longer survival for Stage III versus Stage I/II patients was a function of their earlier mean age at $\mathrm{PCa}$ diagnosis as well as the assumption that survival for these patients would follow the U.S. general population. Rather than report age-adjusted survival estimates, the authors considered it important to have the survival data reflect the finding that Stage III patients are diagnosed at earlier ages relative to Stages I/II.

This study was subject to several limitations. Since PCa patients generally have a good prognosis following diagnosis and therefore long survival times, we did not have information on the date of death for the majority of patients in SEER. This was especially problematic for patients diagnosed at Stages I-III where it was assumed survival for these patients would follow the U.S. general population. This was a conservative assumption as a prior study reported relative survival rates for early stage PCa that were greater than $100 \%$ [9].

In partitioning the survival periods of patients into distinct phases of care, we used methodology similar to a study conducted by Brown and colleagues [7,11]. It should be noted that these studies were conducted in populations of colorectal, breast and lung cancer patients. However, we believe that the application of their methodology to $\mathrm{PCa}$ is appropriate despite the fact that many early stage patients may receive watchful waiting. In a prior study, we found that $\sim 45 \%$ of Stage I patients received active therapy [12]. It may be argued that, for Stage I-IIa patients where a watchful waiting approach is more common, separate initial and continuing care phases may not have been warranted. However, in defining treatment phases we also looked at diagnostic procedures for cancer staging as well as outpatient visits and hospitalizations and, in our exploratory analyses, we found that more early stage $\mathrm{PCa}$ patients used these service types in the first 6 months following cancer diagnosis. Therefore, we considered separate initial and continuing care phases appropriate for early stage PCa. Furthermore, our analyses of healthcare costs for each treatment phase among Stage I patients showed a U-shaped pattern characteristic of patients utilizing a higher degree of medical services both in the months shortly following PCa diagnosis and in the months leading up to death compared to the months defined as continuing care.

The economic analysis was conducted from a payer perspective and includes reimbursement payments made to physicians, facilities and other healthcare professionals for the medical services that were provided to $\mathrm{PCa}$ patients. Other components of the economic burden of $\mathrm{PCa}$ including out-of-pocket spending on direct medical care as well as the indirect costs associated with reduced productivity and lost work time for both caregivers and patients were not included in this study. These cost components have been shown to represent an important proportion of the total cost burden. A study by Chang and colleagues reported monthly costs of $\$ 373, \$ 698$, and $\$ 302$ for absenteeism, short-term disability, and deductibles/copayments, respectively among newly diagnosed cancer (including brain, colorectal, lung, ovarian, pancreatic, prostate, and non-Hodgkin's lymphoma) patients during the first few years following cancer diagnosis [18]. Absenteeism and short-term disability data were not available in the data sources used for the current analysis. However, this may not have contributed greatly to costs related to $\mathrm{PCa}$ as the population was $\geq 65$ years old and not likely to have still been working. It should also be noted that Medicare cost data from calendar years 1991 through 2004 were used for this current study. During this time, Medicare did not cover most prescription drugs. Hence, prescription drug costs were not included in our estimates of direct medical costs.

It is also important to note that our survival estimates for Stage IV patients do not account for the index year of $\mathrm{PCa}$ diagnosis in order to adjust for improvements in prognosis among patients diagnosed in more recent years. A recent study reports that during the early 1990s until 2001, the 5-year relative survival of Stage IV patients has increased from approximately 50\%-60\% [19]. Therefore, this will have the effect of underestimating slightly the economic burden of PCa for recently diagnosed Stage IV patients.

Finally, it should be noted that the estimation of lifetime costs is an area of research that is highly speculative 
[20]. Our analyses are based on the treatment experience and cost profiles of a cohort of patients diagnosed during calendar years 1991 through 2005. It is unlikely that the resource trends and costs of patients diagnosed in 2008 and more recently will exactly mirror the experience of past cohorts. For example, laproscopic and robot-assisted surgical methods are now currently more widely used. Advances have also been made in the treatment of advanced stage PCa. In 2004, it was shown that docetaxel can prolong survival in men with advanced PCa no longer responding to hormone therapy and in 2010, cabazitaxel was approved for use in advanced $\mathrm{PCa}$ after failure with docetaxel. As a result of these treatment advances, the PCa cost estimates reported in this study will probably understate slightly the initial and life-time treatment costs for PCa patients diagnosed today.

\section{Conclusions}

Our analyses offer insight into the magnitude of total and disease-related lifetime costs for patients $\geq 65$ years of age at PCa diagnosis in an era of improved screening and treatment (1991-2002). Long-term estimates are useful for understanding the upper limit of treatment costs that could be avoided should prevention strategies prove effective in reducing incident $\mathrm{PCa}$ cases. Study results indicate that the aggregate lifetime PCa-related burden for 2008 incident cases $\geq 65$ years old is approximately $\$ 4.0$ billion.

\section{Acknowledgements}

This study used the linked SEER-Medicare database. The interpretation and reporting of these data are the sole responsibility of the authors. The authors acknowledge the efforts of the Applied Research Program, National Cancer Institute; the Office of Research, Development and Information, Centers for Medicare and Medicaid Services; Information Management Services, Inc.; and the Surveillance, Epidemiology, and End Results (SEER) Program tumor registries in the creation of the SEER-Medicare database. This research project received funding from GlaxoSmithKline.

\section{Author details \\ ${ }^{1}$ Health Economics, United BioSource Corporation, 185 Dorval Avenue, Montreal, Quebec, H9S 5J9, Canada. ${ }^{2}$ Global Health Outcomes, \\ GlaxoSmithKline, 5 Moore Drive, Research Triangle Park, NC, 27709, USA ${ }^{3}$ Biostatistics and Bioinformatics, Emory University, Rollins School of Public Health, 1518 Clifton Road NE, Atlanta, GA, 30322, USA.}

\section{Authors' contributions}

MES participated in the design and coordination of the study, analysis and interpretation of the data, and the drafting of the manuscript. IP created the study analytic file from the raw data, carried out the statistical analysis, and participated in the revision of the manuscript. LKB participated in the design of the study, analysis and interpretation of the data, and revision of the manuscript. $\mathrm{KJ}$ and $\mathrm{YH}$ participated in the design of the study and analysis and interpretation of the data. All authors read and approved the final manuscript.

\section{Competing interests}

MES, IP, and KJ are full-time employees of United BioSource Corporation (UBC). UBC received funding from GlaxoSmithKline (GSK) for the conduct of this study and the drafting of the manuscript. LKB is an employee of GSK and receives a salary from GSK. YH is a paid consultant for UBC.
Received: 24 October 2011 Accepted: 28 December 2011

Published: 28 December 2011

\section{References}

1. Table I-21, US Prevalence Counts, Invasive Cancers Only, January 1, 2005, Using Different Tumor Inclusion Criteria. In SEER Cancer Statistics Review, 1975-2005. Edited by: Ries L, Melbert D, Krapcho M, et al. Bethesda: National Cancer Institute; 2006.

2. American Cancer Society: Cancer Facts and Figures 2008.

3. National Cancer Institute: Surveillance Epidemiology and End Results (SEER) Stat Fact Sheets: Prostate. Bethesda: National Cancer Institute; 2011.

4. National Cancer Institute: Cancer Trends Progress Report - 2007 Update. Bethesda: National Cancer Institute; 2008.

5. Andriole G, Bostwick D, Brawley O, Gomella L, Marberger M, Tindall D, Breed S, Somerville M, Rittmaster R: Chemoprevention of prostate cancer in men at high risk: rationale and design of the reduction by dutasteride of prostate cancer events (REDUCE) trial. J Urol 2004, 172(4 Pt 1):1314-1317.

6. Thompson IM, Goodman PJ, Tangen CM, Lucia MS, Miller GJ, Ford LG, Lieber MM, Cespedes RD, Atkins JN, Lippman SM, et al: The influence of finasteride on the development of prostate cancer. N Engl J Med 2003, 349(3):215-224.

7. Brown ML, Riley GF, Potosky AL, Etzioni RD: Obtaining long-term disease specific costs of care: application to Medicare enrollees diagnosed with colorectal cancer. Med Care 1999, 37(12):1249-1259.

8. National Cancer Institute: Overview of the SEER Program. Surveillance Epidemiology and End Results Bethesda: National Cancer Institute; 2009.

9. Brenner $H$, Arndt V: Long-term survival rates of patients with prostate cancer in the prostate-specific antigen screening era: population-based estimates for the year 2000 by period analysis. J Clin Oncol 2005, 23(3):441-447.

10. Arias E: United States life tables, 2004. Nat/ Vital Stat Rep 2007, 56(9):1-39.

11. Baker MS, Kessler LG, Urban N, Smucker RC: Estimating the treatment costs of breast and lung cancer. Med Care 1991, 29(1):40-49.

12. Stokes ME, Black L, Benedict A, Roehrborn CG, Albertsen P: Long-term medical-care costs related to prostate cancer: estimates from linked SEER-Medicare data. Prostate Cancer Prostatic Dis 2010.

13. Efron B, Tibshirani R: An Introduction to the Bootstrap New York: Chapman \& Hall; 1993.

14. Riley GF, Potosky AL, Lubitz JD, Kessler LG: Medicare payments from diagnosis to death for elderly cancer patients by stage at diagnosis. Med Care 1995, 33(8):828-841.

15. Lin DW, Porter M, Montgomery B: Treatment and survival outcomes in young men diagnosed with prostate cancer: a Population-based Cohort Study. Cancer 2009, 115(13):2863-2871.

16. Mathers MJ, Roth S, Klinkhammer-Schalke M, Gerken M, Hofstaedter F, Wilm S, Klotz T: Patients with localised prostate cancer ( $\mathrm{t} 1$ - $\mathrm{t} 2)$ show improved overall long-term survival compared to the normal population. J Cancer 2011, 2:76-80.

17. Zeliadt SB, Etzioni R, Ramsey SD, Penson DF, Potosky AL: Trends in treatment costs for localized prostate cancer: the healthy screenee effect. Med Care 2007, 45(2):154-159.

18. Chang S, Long SR, Kutikova L, Bowman L, Finley D, Crown WH, Bennett CL: Estimating the cost of cancer: results on the basis of claims data analyses for cancer patients diagnosed with seven types of cancer during 1999 to 2000. J Clin Oncol 2004, 22(17):3524-3530.

19. Cetin K, Beebe-Dimmer JL, Fryzek JP, Markus R, Carducci MA: Recent time trends in the epidemiology of stage IV prostate cancer in the United States: analysis of data from the Surveillance, Epidemiology, and End Results Program. Urology 2010, 75(6):1396-1404.

20. Brown ML, Riley GF, Schussler N, Etzioni R: Estimating health care costs related to cancer treatment from SEER-Medicare data. Med Care 2002, 40(8 Suppl), IV-104-117.

Pre-publication history

The pre-publication history for this paper can be accessed here: http://www.biomedcentral.com/1472-6963/11/349/prepub

doi:10.1186/1472-6963-11-349

Cite this article as: Stokes et al:: Lifetime economic burden of prostate cancer. BMC Health Services Research 2011 11:349. 